high in aspects of the investigator and clinical research coordinator, but low in the site and sponsor aspects. There were no differences in the satisfaction degree with general characteristics. The satisfaction degree of the acupuncture and moxibustion clinical trials was strong positive correlated with participation benefits $(r=0.595)$ and easy contact with the research team $(\mathrm{r}=0.500)$.

Conclusion: Most subjects showed positive perception and interest in the clinical trials. Future studies should investigate how personal factors influence the satisfaction of subjects, should identify a specific classification criterion, and create a standardized protocol for the clinical trials.

Contact: Hee-Jung Jung, only41@kiom.re.kr

\section{EDUCATION RESEARCH}

\section{P03.01}

Evidence Informed Practice: Acupuncture and Oriental Medicine Faculty and Student Attitudes

Belinda Anderson (1), Roni Evans (2), Ben Kligler (3), Barry Taylor (2), Paul Marantz (3)

(1) Pacific College of Oriental Medicine, New York, NY, USA (2) Northwestern Health Sciences University, Bloomington, $M N$, USA

(3) Albert Einstein College of Medicine, Bronx, NY, USA

Purpose: The NIH National Center for Complementary and Alternative Medicine (NCCAM) awarded a small number of R25 grants to CAM institutions in partnership with researchintensive institutions to develop evidence informed practice (EIP) faculty training and curriculum. Following this model Pacific College of Oriental Medicine (PCOM-NY) has partnered with Albert Einstein College of Medicine (Einstein) and Northwestern Health Sciences University (NWHSU) to undertake a similar project, which is supported by NCCAM funding. PCOM faculty will be trained in EIP using the on-line educational materials developed at NWHSU through their R25 grants. Trained PCOM faculty and administrators, under the guidance of experts from Einstein and NWHSU, will develop EIP curriculum in the Acupuncture and Oriental medicine (AOM) programs. To assess faculty and student research knowledge, support of EIP, and faculty attitudes and interest in this project, pilot surveys instruments were developed and used to generate data for the grant proposal.

Results: The response rates for the faculty $(\mathrm{n}=101)$ and student $(n=201)$ surveys were $87.8 \%$ and $33.4 \%$, respectively. The results from the surveys demonstrated that while students and faculty seemed fairly confident about their information mastery skills, the student's desire for further research training and attitude about the value of EIP ranged from neutral to agree for most questions. Faculty placed a higher value on EIP; with responses to questions ranging from agree to strongly agree. Outcomes from the faculty survey strongly indicated that faculty support the College's endeavor to engage in research, feel they can make a worthwhile contribution, are interested in further research training, and would like to participate in developing EIP curriculum within the AOM program.

Conclusion: This research highlights the fact that introducing EIP curriculum to PCOM will need to be accompanied by culture change in the institution, which was also reported to be a significant factor of the R25 projects.

Contact: Belinda Anderson, banderson@pacificcollege.edu
P03.02

Predictors of Knowledge and Use of Research Literacy Skills Among Students of Acupuncture and Chiropractic Programs

S. Prasad Vinjamury (1), Judith Miller (1)

(1) Southern California University of Health Sciences, Whittier, CA, USA

Purpose: There is a general indifference among CAM students towards the research methods courses developed to teach research literacy. This general apathy calls for constant evaluation of the pedagogy adopted to teach this course. "Research Debates" were adopted at a CAM school with an intention to encourage students' participation in this course as well to help them retain the research literacy skills. We previously assessed and reported the self-perception of students who participated in the research debates as part of this course. The purpose of this paper is: To Identify the predictors of Knowledge and Use of Research Literacy Skills among students of Acupuncture and Chiropractic Programs.

Methods: Sample: All currently enrolled students who passed the Research Methods course and participated in the research debates. Design: Cross-Sectional Study (Survey Design). A questionnaire developed exclusively for this study was administered both in-person and online to collect the data. The survey was anonymous and voluntary in nature.

Results: A total of 171 students responded to the survey. The respondents were predominantly males, chiropractic students, and reported having a bachelor's degree as the highest level of education prior to entering the CAM programs. The results indicated that a majority of students rated that their research literacy skills, which included familiarity with databases and critical appraisal skills, as either outstanding, above average or average. Most respondents also reported that they used these skills at least 1-5 times in clinical internship in the past week. The pedagogy adopted to teach these skills, research debates were also rated either very useful or useful by a majority of these respondents. However, there was no association between the age, gender, program or highest level of education to knowledge of databases or critical appraisal skills.

Conclusion: CAM students perceive their research literacy skills high, but there are no predictors for these perceptions.

Contact: S. Prasad Vinjamury, sivaramavinjamury@scuhs.edu

\section{P03.03}

\section{Core Competencies for Integrative Medicine Fellowship Training Programs}

Melinda Ring (1), Marc Brodsky (2), Tieraona Low Dog (3), Victor Sierpina (4), Michelle Bailey (5), Amy Locke (6), Mikhail Kogan (7), Adam Rindfleisch (8), Robert Saper (9)

(1) Northwestern University, Chicago, IL, USA

(2) Stamford Hospital/Columbia University, Stamford, CT, USA

(3) University of Arizona Center for Integrative Medicine, Tucson, AZ, USA

(4) University of Texas, UTMB Health, Galveston, TX, USA

(5) Duke University, Durham, NC, USA

(6) University of Michigan, Ann Arbor, MI, USA

(7) George Washington Center for Integrative Medicine, Washington, DC, USA

(8) University of Wisconsin, Madison, WI, USA 
(9) Boston Medical Center, Boston University School of Medicine, Boston, MA, USA

Purpose: Integrative Medicine Fellowships are becoming well established in academic health centers. In 2010 the Consortium of Academic Health Centers for Integrative Medicine (CAHCIM) convened a 2-year Task Force to study the characteristics and curricula of existing Fellowships and draft Integrative Medicine Fellowship core competencies.

Methods: The Task Force convened by CAHCIM reviewed the learning objectives and curricula of the 13 existing Integrative Medicine clinical Fellowships. All Fellowship Directors were contacted by email or phone to complete a template of questions regarding program design, curriculum, financial information, educational methods, and required competencies. Through regular conference calls, Task Force members used this survey information, the existing scientific literature, and their own expertise to draft an initial set of competencies using the ACGME categories. Task Force and other CAHCIM members revised the competencies through several monthly conference calls and through email. Oral presentations at professional conferences provided opportunities for public commentary and discussion. The Task Force additionally solicited constructive feedback from Integrative Medicine Fellowship Directors of all existing programs.

Results: Differentiation of competencies for Integrative Medicine Fellows from customary care as described by other medical specialties was most notable in four areas of Medical Knowledge and Patient Care: 1) Pain Management, 2) Nutritional Science, 3) Mind-Body Medicine, and 4) Lifestyle Medicine. Additional Integrative Medicine competencies include characteristics of commonly used evidence-based CAM practices, demographic and epidemiologic patterns of Integrative Medicine use, and relevant legal and ethical issues.

Conclusion: Developing uniform minimum standards for Integrative Medicine clinical Fellowship graduates are necessary to ensure quality training for future leaders in the field. Mastery of these competencies may be fostered by Fellowship programs through innovative training strategies, validated assessment methods, and continued faculty development tailored to the unique strengths and characteristics of each site.

Contact: Marc Brodsky, mbrodsky@stamhealth.org

\section{P03.04}

Surveys of Cancer Patients and Cancer Care Providers Regarding Complementary Therapy Use, Communication and Information Needs

Ngaire King (1), Lynda Balneaves (2), Cynthia Card (3), Jill Nation (3), Thao Nguyen (3), Linda Carlson (3)

(1) University of Calgary, Alberta, Canada

(2) University of British Columbia, Vancouver, British Columbia, Canada

(3) Alberta Health Services, Alberta, Canada

Purpose: In previous surveys, approximately $45 \%$ of cancer patients have reported the use of various Complementary Therapies (CTs). The high prevalence of use creates a need for a closer examination of patient and health care practitioner (HCP) CT use, knowledge, communication, opinions, and beliefs. We sought to compare statistics from the Tom Baker Cancer Centre
(TBCC) in Alberta, Canada, with other published surveys worldwide.

Methods: 481 cancer patients and $100 \mathrm{HCPs}$ were surveyed at the TBCC. Patients were surveyed on their use of CTs, their motivations and barriers to use, patient-practitioner interactions, and their interest in CT resources. HCPs were asked about patient-practitioner interactions, their CAM knowledge and skills, their CAM opinions and beliefs, and their CAM training.

Results: $47 \%(n=227)$ of patients had used CTs since their diagnosis. Patients' top reason for CT use was to improve quality of life $(65 \%)$. The largest barrier to use was uncertainty of the evidence supporting CTs (35.9\%). $80 \%$ of patients had not had a HCP speak to them about CTs and few felt they had received enough CT information (16\%) or support (14\%). $73 \%$ of patients indicated they would utilize a CT education program. $71 \%$ of HCPs had patients report their CAM use. $62.5 \%$ reported asking patients about using CAM. Few practitioners reported being knowledgeable about CAM (18\%) or prepared to address CAM (13\%). HCPs endorsed positive views of CAM, with $66 \%$ believing CAM can benefit conventional medicine. Though only $31 \%$ of practitioners had received any CAM training, $90 \%$ reported interest in receiving CAM education.

Conclusion: CT use is similar at our centre to other surveys worldwide. There is a strong need for patient and practitioner educational resources, enhanced communication about CTs, support for decision making and greater integration of CTs into conventional medicine.

Contact: Linda Carlson, Lcarlso@ucalgary.ca

\section{P03.05}

A Barrier Opener for Personal Insights: Nursing and Medical Students Experiences of Participating in an Experiential Mind-Body Skills Program

Marja van Vliet (1), Mats Jong (2), Miek Jong (3)

(1) Health \& Nutrition/Louis Bolk Institute, Driebergen, Netherlands

(2) Department of Nursing/Mid Sweden University, Sundsvall, Sweden

(3) Department of Nursing/Mid Sweden University and Health \& Nutrition/Louis Bolk Institute, Sundsvall/Driebergen, Sweden/ Netherlands

Purpose: This study aimed to obtain an in-depth understanding on how nursing-, and medical students participating in a MindBody Medicine Skills program experienced participation and what the program has meant to them on a personal and a professional level.

Methods: Based on a qualitative approach, first and second year students were interviewed 3 months after completion of the program. Interviews were analyzed with a qualitative content analysis. As a part of a larger study evaluating the effects on stress, empathy and self-reflection, this qualitative sub-study included 10 nursing students from Mid Sweden University, Sweden, and 10 medical students from University of Utrecht, The Netherlands. The Mind-Body Medicine Skills program was adapted from the program developed at Georgetown University School of Medicine, Washington DC, and included 11 experiential sessions were the students were introduced to different mind-body techniques (mindfulness meditation, guided imagery, bio-feedback etc).

Results: Data analysis is ongoing and more complete analysis will be presented at the conference. In preliminary analysis an 